\title{
Maintenance Overtime Policy with Cumulative Damage
}

\author{
Satoshi Mizutani \\ Department of Media Informatics \\ Aichi University of Technology, Aichi, Japan \\ Corresponding author: mizutani@aut.ac.jp \\ Toshio Nakagawa \\ Department of Business Administration \\ Aichi Institute of Technology, Aichi, Japan \\ E-mail: toshi-nakagawa@aitech.ac.jp
}

(Received March 29, 2017; Accepted September 27, 2017)

\begin{abstract}
We propose an extended maintenance overtime policy for the cumulative damage model where an operating unit suffers some damage due to shocks. It is assumed that the total damage is additive, and the unit fails when the total damage has exceeded a prespecified damage level. It is supposed that we start to observe occurrence of shocks after time $T$, and the unit is replaced at $N$ th $(N=1,2, \ldots)$ shock over time $T$ or at failure, whichever occurs first. That is, we propose a new policy by extending maintenance overtime policy. One example is a rental of system such as industrial equipment with some reservations. For such systems, they should be maintained or replaced at a prespecified number of uses over a scheduled time. For such a model, we obtain the mean time to replacement and the expected costs rate. Further, we discuss about optimal number $N^{*}$ and time $T^{*}$ which minimizes the expected cost rate when shocks occur in a Poisson process. Finally, numerical examples are given, and suitable discussions are made.
\end{abstract}

Keyword - Overtime policy, Replacement policy, Shock model, Cumulative damage

\section{Introduction}

We consider a modified maintenance overtime policy in which an operating unit is replaced at shocks and a damage level. In recent years, equipment management has become more important to complete projects rapidly, safety and accurately. Furthermore, the equipment has become more complexity, and more difficult to check its state by looking the appearance, which includes information unit for software development. We consider therefore that the equipment is replaced at a completion of uses to avoid interruption of work on the way of using cycles. Such a model is called as maintenance overtime policy (Nakagawa and Zhao, 2015). Furthermore, we suppose that the equipment suffers damage at every use, and fails when the total damage has exceeded a prespecified level. Such a model is called as shock and damage model (Nakagawa, 2007). A shock model in which interval times of shocks depend on stochastically the previous shock was proposed in Markovian environment (Cha and Finkelstein, 2013; Eryilmaz, 2016).

We can see many studies of maintenance policies applied stochastic processes such as renewal theory (Barlow and Proschan, 1965; Nakagawa, 2005). The maintenance models that the unit is replaced at a random operating time are studied (Nakagawa, 2014; Chen et al., 2010a; Chen et al., 2010b). Maintenance overtime policies where the unit is replaced at the first time of a completion time of works over planned time have been discussed (Nakagawa and Zhao, 2015; Zhao and Nakagawa, 2013; Zhao et al., 2014). In the model, the unit is not replaced while it is working, because it has much loss cost to stop its work. When the unit is used for several works, we should 
plan a standard time to replace it at the completion of works. So that, replacing working number with shock number, we can consider replacement policies for cumulative damages models. There were also many studies in cumulative damage models (Stallmeyer and Walker, 1968; Bogdanoff and Kozin, 1985; Nakagawa, 2007), and network reliability models under the assumption that the components are subject to shock was introduced (Zarezadeh et al., 2016).

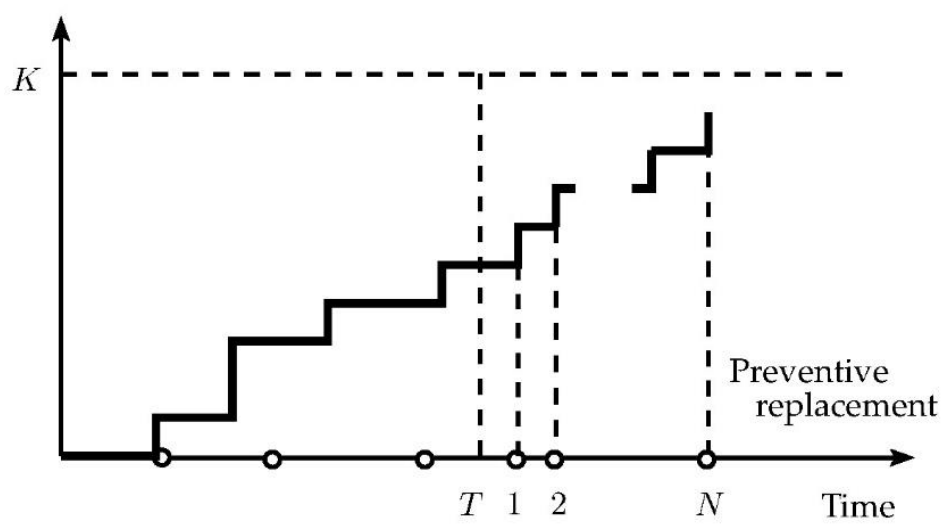

Fig. 1. Process for preventive replacement

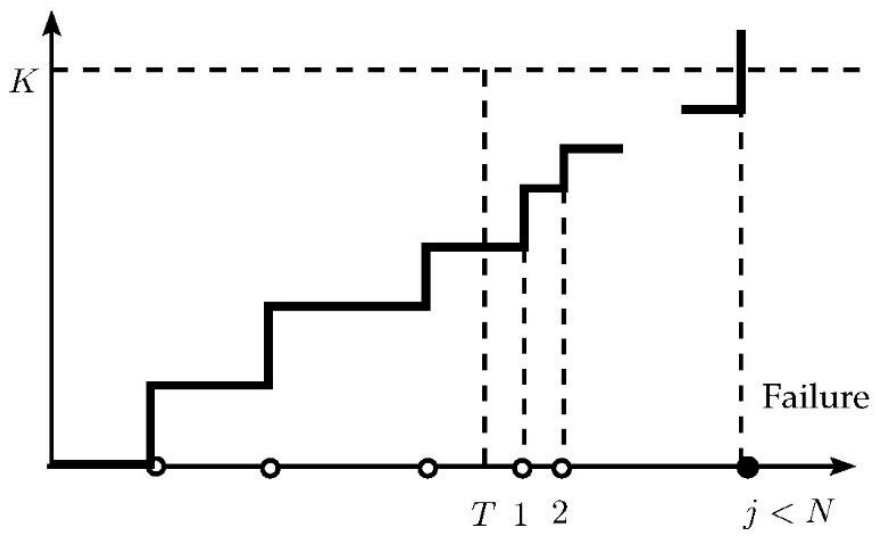

Fig. 2. Process for failure

We propose a maintenance policy of an operating unit which extends the overtime policy to a cumulative damage model: It would be reasonable for the unit to make appropriate policies with scheduled time or number of shocks to maintain or replace it. One example is a rental of equipments with some reservations. For such equipments, they should be maintained or replaced at a prespecified number of uses over a scheduled time. On the other hand, when the possibility of failures before a scheduled time is very low, we do not need to count the number of shocks before this time and can save time. From the above viewpoints, we suppose that the unit is replaced at $N$ th $(N=1,2, \ldots)$ shock over a scheduled time $T$ or at failure, whichever occurs first. Fig. 1 shows that the preventive replacement is done at $N$ th shock over time $T$, and Fig. 2 shows that it is 
replaced at failure. In the figures, horizontal axis presents the process of time, and vertical axis presents the amount of total damage due to shocks. This policy includes several maintenance policies for a cumulative damage model (Nakagawa, 2007).

For the above model, we obtain the expected costs rate and discuss optimal policies which minimize them. Section 2 shows the assumptions and notations, and obtains the mean time to replacement and the expected cost rate. Section 3 discusses optimal number $N^{*}$ and time $T^{*}$ which minimize the expected cost rate when shocks occur in a Poisson process. Section 4 gives numerical examples of optimal $N^{*}$ and $T^{*}$ when each damage is exponential. We investigate several tendencies for suitable parameters in numerical examples. Section 5 takes up a new replacement policy over damage $Z$, and obtains the expected cost rates.

\section{Assumptions}

We make the following assumptions of a replacement policy for the cumulative damage model:

(i) Let $X_{j}$ denote a random variable that denotes a sequence of interval times between successive shocks with an identical distribution $F(t) \equiv \operatorname{Pr}\left\{X_{j} \leq t\right\} \quad(j=1,2, \ldots)$ and finite mean $\mu \equiv \int_{0}^{\infty} \bar{F}(t) d t$, where $\bar{\Phi}(t) \equiv 1-\Phi(\mathrm{t})$ for any function $\Phi(t)$. We define the $j$-fold Stieltjes convolution of $F(t)$, which is denoted by $F^{(j)}(t) \equiv \operatorname{Pr}\left\{X_{1}+X_{2}+\right.$ $\left.\cdots+X_{j} \leq t\right\}(j=1,2, \ldots)$ and $F^{(0)}(t) \equiv 1$ for $t \geq 0$.

(ii) Let $W_{j}$ denote a random variable that denotes the damage produced by the $j$ th shock, where $W_{0} \equiv 0$, with a cumulative distribution $G(t) \equiv \operatorname{Pr}\left\{W_{j} \leq t\right\} \quad(j=1,2, \ldots)$. The $j$-fold Stieltjes convolution of $G(t)$ is defined by $G^{(j)}(t) \equiv \operatorname{Pr}\left\{W_{1}+W_{2}+\cdots+W_{j} \leq t\right\}$ $(j=1,2, \ldots)$ and $G^{(0)}(t) \equiv 1$ for $t \geq 0$.

(iii) Let $N(t)$ denote a random variable which is the total number of shocks up to time $t$. Then, we define a random variable $Z(t)$ as follows:

$$
Z(t) \equiv \sum_{j=0}^{N(t)} W_{j},
$$

which represents the total damage at time $t$. The unit fails when the total damage has exceeded a prespecified damage level $K(0<K<\infty)$.

(iv) The unit is replaced at failure or at $N$ th $(N=1,2, \ldots)$ shock over time $T(0 \leq T<\infty)$, whichever occurs first (see, Fig. 1 and Fig. 2).

(v) Cost $c_{F}$ is a replacement cost when the unit fails, and cost $c_{N}$ is a replacement cost when the unit is replaced at $N$ th shock over time $T$, where $c_{F}>c_{N}$.

From the above assumptions, we obtain the mean time to replacement and the expected cost rate analytically as follows: The probability that the unit is replaced before failure at shock $N$ over time $T$ is 


$$
\begin{aligned}
& \sum_{j=0}^{\infty} G^{(j+N)}(K) \int_{0}^{T}\left\{\int_{T-t}^{\infty}\left[\int_{u}^{\infty} \mathrm{d} F^{(N-1)}(v-u)\right] \mathrm{d} F(u)\right\} \mathrm{d} F^{(j)}(t) \\
& =\sum_{j=0}^{\infty}\left[F^{(j)}(T)-F^{(j+1)}(T)\right] G^{(j+N)}(K)
\end{aligned}
$$

and the probability that it is replaced at failure is

$$
\sum_{j=0}^{\infty} F^{(j+1)}(T)\left[G^{(j)}(K)-G^{(j+1)}(K)\right]+\sum_{j=0}^{\infty}\left[F^{(j)}(T)-F^{(j+1)}(T)\right]\left[G^{(j)}(K)-G^{(j+N)}(K)\right]
$$

where $(1)+(2)=1$. The mean time to replacement is

$$
\begin{aligned}
& \sum_{j=0}^{\infty} G^{(j+N)}(K) \int_{0}^{T}\left\{\int_{T-t}^{\infty}\left[\int_{u}^{\infty}(t+v) \mathrm{d} F^{(N-1)}(v-u)\right] \mathrm{d} F(u)\right\} \mathrm{d} F^{(j)}(t) \\
& \quad+\sum_{j=0}^{\infty}\left[G^{(j)}(K)-G^{(j+1)}(K)\right] \int_{0}^{T} t \mathrm{~d} F^{(j+1)}(t) \\
& \quad+\sum_{j=0}^{\infty} \sum_{i=0}^{N-1}\left[G^{(j+i)}(K)-G^{(j+i+1)}(K)\right] \int_{0}^{T}\left\{\int_{T-t}^{\infty}\left[\int_{u}^{\infty}(t+v) \mathrm{d} F^{(i)}(v-u)\right] \mathrm{d} F(u)\right\} \mathrm{d} F^{(j)}(t) \\
& =\sum_{j=0}^{\infty} G^{(j+N)}(K) \int_{0}^{T}\left(\int_{T-t}^{\infty}\left\{\int_{u}^{\infty}\left[1-F^{(N-1)}(v-u)\right] \mathrm{d} v\right\} \mathrm{d} F(u)\right) \mathrm{d} F^{(j)}(t) \\
& \quad+\sum_{j=0}^{\infty} \sum_{i=0}^{N-1}\left[G^{(j+i)}(K)-G^{(j+i+1)}(K)\right] \int_{0}^{T}\left(\int_{T-t}^{\infty}\left\{\int_{u}^{\infty}\left[1-F^{(i)}(v-u)\right] \mathrm{d} v\right\} \mathrm{d} F(u)\right) \mathrm{d} F^{(j)}(t) \\
& \quad+\mu \sum_{j=0}^{\infty} F^{(j)}(T) G^{(j)}(K) \\
& =\mu \sum_{j=0}^{\infty}\left[F^{(j)}(T)-F^{(j+1)}(T)\right] \sum_{i=0}^{N+j-1} G^{(i)}(K) .
\end{aligned}
$$

Therefore, the expected cost rate is, from (1) and (3),

$$
C_{1}(N, T)=\frac{c_{F}-\left(c_{F}-c_{N}\right) \sum_{j=0}^{\infty}\left[F^{(j)}(T)-F^{(j+1)}(T)\right] G^{(j+N)}(K)}{\mu \sum_{j=0}^{\infty}\left[F^{(j)}(T)-F^{(j+1)}(T)\right] \sum_{i=0}^{N+j-1} G^{(i)}(K)} .
$$

When the unit is replaced only at $N$ th shock,

$$
C_{1}(N) \equiv \lim _{T \rightarrow 0} C_{1}(N, T)=\frac{c_{F}-\left(c_{F}-c_{N}\right) G^{(N)}(K)}{\mu \sum_{j=0}^{N-1} G^{(j)}(K)} \quad(N=1,2, \ldots)
$$


which agrees with (3.20) of (Nakagawa, 2007). When the unit is replaced only at the first shock over time $T$ is

$$
C_{1}(T) \equiv C_{1}(1, T)=\frac{c_{F}-\left(c_{F}-c_{N}\right) \sum_{j=0}^{\infty}\left[F^{(j)}(T)-F^{(j+1)}(T)\right] G^{(j+1)}(K)}{\mu \sum_{j=0}^{\infty}\left[F^{(j)}(T)-F^{(j+1)}(T)\right] \sum_{i=0}^{j} G^{(i)}(K)}
$$

which agrees with (3.50) of (Nakagawa, 2007).

\section{Optimal Replacement Policies}

When $F(t)=1-e^{-\lambda t}$, the expected cost rate in (4) is

$$
\frac{C_{1}(N, T)}{\lambda}=\frac{c_{F}-\left(c_{F}-c_{N}\right) \sum_{j=0}^{\infty}\left[(\lambda T)^{j} / j !\right] \mathrm{e}^{-\lambda T} G^{(j+N)}(K)}{\sum_{j=0}^{\infty}\left[(\lambda T)^{j} / j !\right] \mathrm{e}^{-\lambda T} \sum_{i=0}^{N+j-1} G^{(i)}(K)} .
$$

It is assumed that

$$
Q(N)=\frac{G^{(N)}(K)-G^{(N+1)}(K)}{G^{(N)}(K)} \quad(N=0,1,2, \ldots)
$$

increases strictly with $N$ from $\bar{G}(K)$ to 1 . Noting that $Q(N)$ represents the probability that the unit fails at shock $N+1$, given that it has not failed until shock $N$, this assumption would be reasonable in actual fields.

\subsection{Optimal $N^{*}$}

We find optimal $N^{*}$ to minimize $C_{1}(N)$ in (5). Forming the inequality $C_{1}(N+1)-C_{1}(N) \geq 0$,

$$
Q(N) \sum_{j=0}^{N-1} G^{(j)}(K)+G^{(N)}(K) \geq \frac{c_{F}}{c_{F}-c_{N}}
$$

Where

$$
\begin{aligned}
& Q(N, T) \equiv \frac{\sum_{j=0}^{\infty}\left[(\lambda T)^{j} / j !\right]\left[G^{(j+N)}(K)-G^{(j+N+1)}(K)\right]}{\sum_{j=0}^{\infty}\left[(\lambda T)^{j} / j !\right] G^{(j+N)}(K)}, \\
& Q(N) \equiv Q(N, 0),
\end{aligned}
$$

$Q(T) \equiv Q(1, T)=\frac{\sum_{j=0}^{\infty}\left[(\lambda T)^{j} / j !\right]\left[G^{(j+1)}(K)-G^{(j+2)}(K)\right]}{\sum_{j=0}^{\infty}\left[(\lambda T)^{j} / j !\right] G^{(j+1)}(K)}$. 
Note that $Q(N, T)$ increases strictly with $N$ from $Q(T)$ to 1 , and increases strictly with $T$ from $Q(N)$ to 1 from Appendix. Thus, the left-hand side of (9) increases strictly with $N$ from 1 to $1+M(K)$, where $M(K) \equiv \sum_{i=1}^{\infty} G^{(i)}(K)$, and represents the expected number of shocks until failure. Therefore, if $M(K)$ is greater than $\mathrm{c}_{N} /\left(c_{F}-c_{N}\right)$, then there exists a finite and unique minimum $N^{*}\left(1 \leq N^{*}<\infty\right)$ which satisfies (8).

\subsection{Optimal $T^{*}$}

We find optimal $T^{*}$ to minimize $C_{1}(T)$ in (6). Differentiating $C_{1}(T)$ with respect to $T$ and setting it equal to zero,

$Q(T) \sum_{j=0}^{\infty} \frac{(\lambda T)^{j}}{j !} e^{-\lambda T} \sum_{i=0}^{j} G^{(i)}(K)+\sum_{j=0}^{\infty} \frac{(\lambda T)^{j}}{j !} e^{-\lambda T} G^{(j+1)}(K)=\frac{c_{F}}{c_{F}-c_{N}}$

whose left-hand side increases strictly with $T$ to $1+M(K)$, because $Q(T)$ increases strictly with $T$ from $\left[G(K)-G^{(2)}(K)\right] / G(K)$ to 1 . Thus, if $M(K)>c_{N} /\left(c_{F}-c_{N}\right)$, then there exists a finite and unique $T^{*}\left(0 \leq T^{*}<\infty\right)$ which satisfies $(9)$, and the resulting cost rate is

$$
C\left(T^{*}\right)=\frac{\left(c_{F}-c_{N}\right) Q\left(T^{*}\right)}{\mu} .
$$

\subsection{Optimal $N_{0}^{*}$ and $T_{0}^{*}$}

When $F(t)=1-e^{-\lambda t}, Q(N)$ increases strictly with $N$ to 1 and $M(K)>c_{N} /\left(c_{F}-c_{N}\right)$, we find optimal $N_{0}^{*}$ which minimize $C_{1}(N, T)$ in (7) for given $T$, and optimal $T_{0}^{*}$ which minimize $C_{1}(N, T)$ for given $N$. Furthermore, we discuss about both optimal $N_{0}^{*}$ and $T_{0}^{*}$ which minimize $C_{1}(N, T)$ simultaneously.

First, we find optimal $N_{0}^{*}$ to minimizes $C_{1}(N, T)$ for fixed $T(0 \leq T<\infty)$. Forming the inequality $C_{1}(N+1, T)-C_{1}(N, T) \geq 0$,

$Q(N, T) \sum_{j=0}^{\infty} \frac{(\lambda T)^{j}}{j !} e^{-\lambda T} \sum_{i=0}^{N+j-1} G^{(i)}(K)+\sum_{j=0}^{\infty} \frac{(\lambda T)^{j}}{j !} e^{-\lambda T} G^{(j+N)}(K) \geq \frac{c_{F}}{c_{F}-c_{N}}$

whose left-hand side increases strictly with $N$ to $1+M(K)$. Thus, there exists a finite and unique minimum $N_{0}^{*}\left(1 \leq N_{0}^{*}<\infty\right)$ which satisfies (14).

Furthermore, letting $L(N, T)$ be the left-hand side of $(14), L(N, T)$ increases strictly with $T$ from

$L(N, 0)=Q(N) \sum_{j=0}^{N-1} G^{(j)}(K)+G^{(N)}(K)$ 
which agrees with the left-hand side of (9). Thus, $N_{0}^{*}$ decreases with $T$ from $N^{*}$ given in (9), and $1 \leq N_{0}^{*} \leq N^{*}$. In addition, because $L(1, T)$ agrees with the left-hand side of (12), if $T \geq T^{*}$ given in (12), then $N_{0}^{*}=1$, and conversely, if $T<T^{*}$ then $N_{0}^{*} \geq 2$.

Next, we find optimal $T_{0}^{*}$ to minimize $C(N, T)$ for fixed $N(1 \leq N<\infty)$. Differentiating $C_{1}(N, T)$ with respect to $T$ and setting it equal to zero,

$Q(N, T) \sum_{j=0}^{\infty} \frac{(\lambda T)^{j}}{j !} e^{-\lambda T} \sum_{i=0}^{N+j-1} G^{(i)}(K)+\sum_{j=0}^{\infty} \frac{(\lambda T)^{j}}{j !} e^{-\lambda T} G^{(j+N)}(K)=\frac{c_{F}}{c_{F}-c_{N}}$

whose left-hand side agrees with $L(N, T)$ and increases strictly with $T$ from $L(N, 0)$ given in (9) to $L(N, \infty) \geq M(K)$. Thus, because

$$
L\left(N^{*}, T\right) \geq L\left(N^{*}, 0\right) \geq \frac{c_{F}}{c_{F}-c_{N}}
$$

$T_{0}^{*}=0$, i.e., optimal policy which minimizes $C_{1}(N, T)$ is $N_{0}^{*}=N^{*}$ given in (9) and $T_{0}^{*}=0$. Therefore, replacement with shock $N$ is better than replacement overtime when both replacement costs are the same.

Table 1. Optimal $N_{0}^{*}$ when $\omega K=10$

\begin{tabular}{c|ccccccc}
\hline \multirow{2}{*}{$c_{F} / c_{N}$} & \multicolumn{8}{|c}{$\lambda T$} \\
\cline { 2 - 8 } & 0 & 1 & 2 & 3 & 4 & 5 & 10 \\
\hline \hline 5 & 6 & 5 & 4 & 3 & 2 & 1 & 1 \\
\hline 10 & 5 & 4 & 3 & 2 & 1 & 1 & 1 \\
\hline 20 & 4 & 3 & 2 & 1 & 1 & 1 & 1 \\
\hline 30 & 4 & 3 & 2 & 1 & 1 & 1 & 1 \\
\hline 40 & 4 & 2 & 1 & 1 & 1 & 1 & 1 \\
\hline 50 & 4 & 2 & 1 & 1 & 1 & 1 & 1 \\
\hline & $N^{*}$ & & & & & &
\end{tabular}

Table 2. Optimal $N_{0}^{*}$ when $\omega K=20$

\begin{tabular}{c|ccccccc}
\hline \multirow{2}{*}{$c_{F} / c_{N}$} & \multicolumn{7}{c}{$\lambda T$} \\
\cline { 2 - 8 } & 0 & 1 & 2 & 3 & 4 & 5 & 10 \\
\hline \hline 5 & 13 & 12 & 11 & 10 & 9 & 8 & 2 \\
\hline 10 & 12 & 10 & 9 & 8 & 7 & 6 & 1 \\
\hline 20 & 10 & 9 & 8 & 7 & 6 & 5 & 1 \\
\hline 30 & 10 & 9 & 7 & 6 & 5 & 4 & 1 \\
\hline 40 & 10 & 8 & 7 & 6 & 5 & 3 & 1 \\
\hline 50 & 9 & 8 & 7 & 6 & 4 & 3 & 1 \\
\hline & $N^{*}$ & & & & & &
\end{tabular}


International Journal of Mathematical, Engineering and Management Sciences

Vol. 3, No. 2, 123-135, 2018

https://dx.doi.org/10.33889/IJMEMS.2018.3.2-010

Furthermore, if $N \geq N^{*}$, then $T_{0}^{*}=0$, and conversely, if $N \leq N^{*}-1$, then $L(N, 0)<c_{F} /$ $\left(c_{F}-c_{N}\right)$ and there exists a finite and unique $T_{0}^{*}\left(0<T_{0}^{*}<\infty\right)$ which satisfies $(16)$.

\section{Numerical Examples}

We give numerical examples when $F(t)=1-e^{-\lambda t}$ and $G(x)=1-e^{-\omega x}$. Then, $M(K)=\omega K$,

$Q(N)=\frac{(\omega K)^{N} / N !}{\sum_{j=N}^{\infty}\left[(\omega K)^{j} / j !\right]}$,

increases strictly with $N$ from $\omega K /\left(e^{\omega K}-1\right)$ to 1 ,

$$
Q(T)=\frac{\sum_{j=0}^{\infty}\left[(\lambda T)^{j} / j !\right]\left[(\omega K)^{j+1} /(j+1) !\right]}{\sum_{j=0}^{\infty}\left[(\lambda T)^{j} / j ! \sum_{i=j+1}^{\infty}\left[(\omega K)^{i} / i !\right]\right.},
$$

increases strictly with $T$ from $\omega K /\left(e^{\omega K}-1\right)$ to 1 , and

$$
Q(N, T)=\frac{\sum_{j=0}^{\infty}\left[(\lambda T)^{j} / j !\right]\left[(\omega K)^{j+N} /(j+N) !\right]}{\sum_{j=0}^{\infty}\left[(\lambda T)^{j} / j !\right] \sum_{i=j+N}^{\infty}\left[(\omega K)^{i} / i !\right]},
$$

increases strictly with $N$ from $Q(T)$ to 1 and increases strictly with $T$ from $Q(N)$ to 1 .

\begin{tabular}{|c|c|c|c|c|c|c|}
\hline \multirow[t]{2}{*}{$c_{F} / c_{N}$} & \multicolumn{6}{|c|}{$\omega K$} \\
\hline & 5 & 10 & 15 & 20 & 25 & 30 \\
\hline 5 & 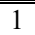 & 3 & 6 & $\begin{array}{lll}10 \\
\end{array}$ & 13 & 17 \\
\hline 10 & 1 & 2 & 5 & 8 & 12 & 15 \\
\hline 20 & 1 & 1 & 4 & 7 & 10 & 14 \\
\hline 30 & 1 & 1 & 3 & 6 & 10 & 13 \\
\hline 40 & 1 & 1 & 3 & 6 & 9 & 13 \\
\hline 50 & 1 & 1 & 3 & 6 & 9 & 12 \\
\hline
\end{tabular}

Table 3. Optimal $N_{0}^{*}$ when $\lambda T=5$

Table 4. Optimal $\lambda T_{0}^{*}$ when $\omega K=10$

\begin{tabular}{c|cccccc}
\hline \multirow{2}{*}{$c_{F} / c_{N}$} & \multicolumn{7}{c}{$N$} \\
\cline { 2 - 7 } & 1 & 2 & 3 & 4 & 5 & 6 \\
\hline \hline 5 & 4.7 & 3.7 & 2.6 & 1.6 & 0.6 & 0.0 \\
\hline 10 & 3.4 & 2.4 & 1.4 & 0.5 & 0.0 & 0.0 \\
\hline 20 & 2.5 & 1.6 & 0.7 & 0.0 & 0.0 & 0.0 \\
\hline 30 & 2.1 & 1.2 & 0.4 & 0.0 & 0.0 & 0.0 \\
\hline 40 & 1.9 & 1.0 & 0.2 & 0.0 & 0.0 & 0.0 \\
\hline 50 & 1.7 & 0.8 & 0.0 & 0.0 & 0.0 & 0.0 \\
\hline & $\lambda T^{*}$ & & & & &
\end{tabular}


Table 5. Optimal $\lambda T_{0}^{*}$ when $\omega K=20$

\begin{tabular}{c|cccccc}
\hline \multirow{2}{*}{$c_{F} / c_{N}$} & \multicolumn{7}{|c}{$N$} & & & \\
\cline { 2 - 7 } & 1 & 2 & 3 & 4 & 5 & 6 \\
\hline \hline 5 & 10.9 & 9.9 & 8.9 & 8.0 & 7.0 & 2.3 \\
\hline 10 & 9.0 & 8.1 & 7.2 & 6.3 & 5.4 & 0.9 \\
\hline 20 & 7.7 & 6.8 & 6.0 & 5.1 & 4.2 & 0.0 \\
\hline 30 & 7.1 & 6.2 & 5.3 & 4.5 & 3.6 & 0.0 \\
\hline 40 & 6.7 & 5.8 & 5.0 & 4.1 & 3.3 & 0.0 \\
\hline 50 & 6.4 & 5.5 & 4.7 & 3.9 & 3.0 & 0.0 \\
\hline & $\lambda T^{*}$ & & & & &
\end{tabular}

Table 6. Optimal $\lambda T_{0}^{*}$ when $N=5$

\begin{tabular}{c|cccccc}
\hline \multirow{2}{*}{$c_{F} / c_{N}$} & \multicolumn{7}{|c}{$\omega K$} \\
\cline { 2 - 7 } & 5 & 10 & 15 & 20 & 25 & 30 \\
\hline \hline 5 & 0.0 & 0.6 & 3.7 & 7.0 & 10.4 & 13.9 \\
\hline 10 & 0.0 & 0.0 & 2.4 & 5.4 & 8.5 & 11.8 \\
\hline 20 & 0.0 & 0.0 & 1.5 & 4.2 & 7.1 & 10.2 \\
\hline 30 & 0.0 & 0.0 & 1.0 & 3.6 & 6.5 & 9.5 \\
\hline 40 & 0.0 & 0.0 & 0.8 & 3.3 & 6.0 & 9.0 \\
\hline 50 & 0.0 & 0.0 & 0.6 & 3.0 & 5.7 & 8.6 \\
\hline
\end{tabular}

Table 1 presents optimal $N_{0}^{*}$ when $\omega K=10$ for $c_{F} / c_{N}$ and $\lambda T$, and when $\lambda T=0, N_{0}^{*}=N^{*}$. For example, when $c_{F} / c_{N}=20$ and $\lambda T=2, N_{0}^{*}=2$ and we should replace the unit at 2 nd shock over time $T$ before failure. We can see that $N_{0}^{*}$ decreases with $c_{F} / c_{N}$ and decreases with $\lambda T$ from $N^{*}$ to 1 . This indicates that if replacement cost $c_{F}$ of failure is large, then we should replace the unit early to avoid its failure. Furthermore, if the expected number $\lambda T$ of shocks until time $T$ is large, then we should replace the unit early.

Table 2 presents optimal $N_{0}^{*}$ when $\omega K=20$ for $c_{F} / c_{N}$ and $\lambda T$. We can see the same tendencies with Table 1 , and $N_{0}^{*}$ is large as $\omega K$ is large. This indicates that if $\omega K$ become large, then the risk of failure decreases and we should replace the unit late. For example, when $\omega K=20$ is twice of one in Table 1, optimal $N_{0}^{*}$ in Table 2 become more than twice of ones in Table 1.

Table 3 presents optimal $N_{0}^{*}$ when $\lambda T=5$ for $c_{F} / c_{N}$ and $\omega K$. This indicates that we should replace the unit late when $\omega K$ is large, because $\omega K$ means the expected number of shocks to failure. It is of interest that $N_{0}^{*}$ increase almost linearly with $\omega K$.

Table 4 presents optimal $\lambda T_{0}^{*}$ when $\omega K=10$ for $c_{F} / c_{N}$ and $N$, and when $N=1, \lambda T_{0}^{*}=\lambda T^{*}$ given (12). We can see that $\lambda T_{0}^{*}$ decreases with $c_{F} / c_{N}$ and also decreases with $N$ from $\lambda T^{*}$ to 0 . This indicates that if replacement $\operatorname{cost} c_{F}$ of failure is large, then we should replace the unit early to avoid its failure. If $c_{F} / c_{N}$ and $N$ are large, optimal $\lambda T^{*}=0$. This means that the unit is replaced only at shock $N$ before failure. 
Table 5 presents optimal $\lambda T_{0}^{*}$ when $\omega K=20$ for $c_{F} / c_{N}$ and $N$. We can see the same tendencies with Table 4 , and $\lambda T_{0}^{*}$ is large as $\omega K$ is large. This indicates that if the expected number $\omega K$ of shocks is large, then we should replace the unit late to avoid its failure. It is of interest that $\lambda T_{0}^{*}$ decreases almost linearly with $N$ from $N=1$ to 5 , and decreases drastically from $N=5$ to 6 , because if $N$ is large, then the risk of failures becomes large and we should replacement the unit much early.

Table 6 presents optimal $\lambda T_{0}^{*}$ when $N=20$ for $c_{F} / c_{N}$ and $\omega K$. We can see that $\lambda T_{0}^{*}$ increases with $\omega K$. This indicates that if the prespecified damage level $\omega K$ is large, then we should set $T$ large to avoid the frequent preventive replacement. It is of interest that $\lambda T_{0}^{*}$ becomes 0 when $\omega K$ is small. Further we can see that $\lambda T_{0}^{*}$ decreases with $c_{F} / c_{N}$. This is the same tendency with Table 4 and Table 5.

\section{Replacement Over damage $Z$}

Suppose that the unit is replaced at $N$ th $(N=1,2, \ldots)$ shock over level $Z(0 \leq Z<K)$ or at failure, whichever occurs first.

The probability that the unit is replaced before failure at shock $N$ over level $Z$ is

$\sum_{j=0}^{\infty} \int_{0}^{Z}\left[\int_{Z-x}^{K-x} G^{(N-1)}(K-x-y) \mathrm{d} G(y)\right] \mathrm{d} G^{(j)}(x)$

and the probability that it is replaced at failure is

$\sum_{j=0}^{\infty} \int_{0}^{Z} \bar{G}(K-x) \mathrm{d} G^{(j)}(x)+\sum_{j=0}^{\infty} \int_{0}^{Z}\left\{\int_{Z-x}^{K-x}\left[1-G^{(N-1)}(K-x-y)\right] \mathrm{d} G(y)\right\} \mathrm{d} G^{(j)}(x)$,

where $(20)+(21)=1$. The expected number of shocks until replacement is

$$
\begin{aligned}
& \sum_{j=0}^{\infty}(j+N) \int_{0}^{Z}\left[\int_{Z-x}^{K-x} G^{(N-1)}(K-x-y) \mathrm{d} G(y)\right] \mathrm{d} G^{(j)}(x)+\sum_{j=0}^{\infty}(j+1) \int_{0}^{Z} \bar{G}(K-x) \mathrm{d} G^{(j)}(x) \\
& +\sum_{j=0}^{\infty} \int_{0}^{Z}\left\{\sum_{i=0}^{N-2}(j+i+2) \int_{Z-x}^{K-x}\left[G^{(i)}(K-x-y)-G^{(i+1)}(K-x-y)\right] \mathrm{d} G(y)\right\} \mathrm{d} G^{(j)}(x) \\
& =\sum_{j=0}^{\infty}\left\{G^{(j)}(Z)+\int_{0}^{Z}\left[\sum_{i=0}^{N-2} \int_{Z-x}^{K-x} G^{(i)}(K-x-y) \mathrm{d} G(y)\right] \mathrm{d} G^{(j)}(x)\right\},
\end{aligned}
$$

and so that, the mean time to replacement is

$$
\mu \sum_{j=0}^{\infty}\left\{G^{(j)}(Z)+\int_{0}^{Z}\left[\sum_{i=0}^{N-2} \int_{Z-x}^{K-x} G^{(i)}(K-x-y) \mathrm{d} G(y)\right] \mathrm{d} G^{(j)}(x)\right\} .
$$


Therefore, the expected cost rate is

$$
C_{2}(N, Z)=\frac{c_{F}-\left(c_{F}-c_{N}\right) \sum_{j=0}^{\infty} \int_{0}^{Z}\left[\int_{Z-x}^{K-x} G^{(N-1)}(K-x-y) \mathrm{d} G(y)\right] \mathrm{d} G^{(j)}(x)}{\mu \sum_{j=0}^{\infty}\left\{G^{(j)}(Z)+\int_{0}^{Z}\left[\sum_{i=0}^{N-2} \int_{Z-x}^{K-x} G^{(i)}(K-x-y) \mathrm{d} G(y)\right] \mathrm{d} G^{(j)}(x)\right\}} .
$$

When the unit is replaced only at $N$ th shock,

$$
C_{2}(N) \equiv C_{2}(N, 0)=\frac{c_{F}-\left(c_{F}-c_{N}\right) G^{(N)}(K)}{\mu \sum_{j=0}^{N-1} G^{(j)}(K)}
$$

which agrees with (5). When the unit is replaced only at the first shock over damage $Z$,

$$
C_{2}(Z) \equiv C_{2}(1, Z)=\frac{c_{F}-\left(c_{F}-c_{N}\right) \sum_{j=0}^{\infty} \int_{0}^{Z}[G(K-x)-G(Z-x)] \mathrm{d} G^{(j)}(x)}{\mu \sum_{j=0}^{\infty} G^{(j)}(Z)}
$$

which agrees with (3.56) of (Nakagawa, 2007).

We could make similar discussions used in Section 3 and derive optimal policies which minimize the expected cost rates analytically, and give numerical examples.

\section{Conclusions}

We have proposed the extended replacement policy for the cumulative damage model over the planned replacement time in which the unit is replaced at $N$ th completion of shocks over time $T$. We have obtained the expected cost rates, and discussed both optimal $T^{*}$ and $N^{*}$ which minimize them analytically and numerically. It has been shown that the replacement with shock $N$ is better than replacement overtime when both replacement costs are the same. Furthermore, we have obtained that if replacement cost of failure is large, then we should replace the unit early to avoid its failure. As another extended policy, we have proposed the replacement in which the unit is replaced at $N$ th completion of shocks over damage level $Z$ and obtained the expected cost rate.

As a future work, we should modify this model more realistically for equipment management. For example, we could consider maintenance policies where equipments are replaced at a random time over planned time. Another example would be given when the number of shocks over time is random. These formulations and results would be applied to real systems such as management projects to develop information systems effectively by suitable modifications. 
International Journal of Mathematical, Engineering and Management Sciences

Vol. 3, No. 2, 123-135, 2018

https://dx.doi.org/10.33889/IJMEMS.2018.3.2-010

\section{Reference}

Barlow, R. E., \& Proschan, F. (1965). Mathematical theory of reliability. John Wiley \& Sons, New York.

Bogdanoff, J. L., \& Kozin, F. (1985). Probabilistic models of cumulative damage. John Wiley \& Sons, New York.

Cha, J. H., \& Finkelstein, M. (2013). On history-dependent shock models. Operations Research Letters, 41(3), 232-237.

Chen, M., Mizutani, S., \& Nakagawa, T. (2010a). Random and age replacement policies. International Journal of Reliability Quality and Safety Engineering, 17(1), 27-39.

Chen, M., Nakamura, S., \& Nakagawa, T. (2010b). Replacement and preventive maintenance models with random working times. IEICE Transactions on Fundamentals, E93-A(2), 500-507.

Eryilmaz, S. (2016). Discrete time shock models in a Markovian environment, IEEE Transactions on Reliability, 65(1), 141-146.

Nakagawa, T. (2005). Maintenance theory of reliability. Springer-Verlag, London.

Nakagawa, T. (2007). Shock and damage models in reliability theory. Springer-Verlag, London.

Nakagawa, T. (2014). Random maintenance policies. Springer-Verlag, London.

Nakagawa, T., \& Zhao, X. (2015). Maintenance overtime policies in reliability theory. Spring-Verlag, London.

Stallmeyer, J. E., \& Walker, W. H. (1968). Cumulative damage theories and application. Journal of the Structual Division, 94(12), 2739-2750.

Zarezadeh, S., Ashrafi, S., \& Asadi, M. (2016). A shock model based approach to network reliability, IEEE Transactions on Reliability, 65(2), 992-1000.

Zhao, X., \& Nakagawa, T. (2013). Optimal periodic and random inspection with first, last, and overtime policies. International Journal of Systems Science, 46(9), 1648-1660.

Zhao, X., Qian, C., \& Nakamura, S., (2014). Age and periodic replacement with overtime policies. International Journal of Reliability, Quality and Safety Engineering, 21(4), 1450016.

\section{Appendix}

When $Q(N)$ increases strictly with $N(N=0,1,2, \ldots)$ to 1 ,

$Q(N, T)=\frac{\sum_{j=0}^{\infty}\left[(\lambda T)^{j} / j !\right]\left[G^{(j+N+1)}-G^{(j+N+2)}(K)\right]}{\sum_{j=0}^{\infty}\left[(\lambda T)^{j} / j !\right] G^{(j+N+1)}(K)}$

increases strictly with $N$ from $Q(T)$ to 1 and increases strictly with $T$ from $Q(N)$ to 1 .

Proof. First, note that for any $N_{1}>0$, 


$$
\begin{aligned}
\lim _{N \rightarrow \infty} \frac{\sum_{j=0}^{N_{1}}\left[(\lambda T)^{j} / j !\right]\left[G^{(j+N)}-G^{(j+N+1)}(K)\right]}{\sum_{j=0}^{N_{1}}\left[(\lambda T)^{j} / j !\right] G^{(j+N+1)}(K)} & =\lim _{N \rightarrow \infty} \frac{G^{\left(N_{1}+N\right)}(K)-G^{\left(N_{1}+N+1\right)}(K)}{G^{\left(N_{1}+N\right)}(K)} \\
& =\lim _{N \rightarrow \infty} Q(N)=1,
\end{aligned}
$$

and

$$
\begin{aligned}
\lim _{T \rightarrow \infty} \frac{\sum_{j=0}^{N_{1}}\left[(\lambda T)^{j} / j !\right]\left[G^{(j+N)}-G^{(j+N+1)}(K)\right]}{\sum_{j=0}^{N_{1}}\left[(\lambda T)^{j} / j !\right] G^{(j+N)}(K)} & =\frac{G^{\left(N_{1}+N\right)}(K)-G^{\left(N_{1}+N+1\right)}(K)}{G^{\left(N_{1}+N\right)}(K)} \\
& =Q\left(N_{1}+N\right)
\end{aligned}
$$

which follows that $\lim _{N \rightarrow \infty} Q(N, T)=\lim _{T \rightarrow \infty} Q(N, T)=1$ because $N_{1}$ is arbitrary.

Next, because $Q(N, T)$ is rewritten as

$$
Q(N, T)=\frac{\sum_{j=N}^{\infty}\left[(\lambda T)^{j-N} /(j-N) !\right]\left[G^{(j)}(K)-G^{(j+1)}(K)\right]}{\sum_{j=N}^{\infty}\left[(\lambda T)^{j-N} /(j-N) !\right] G^{(j)}(K)},
$$

from $Q(N+1, T)-Q(N, T)$,

$$
\begin{aligned}
& \left.G^{(N)}(K) \sum_{j=N}^{\infty} \frac{(\lambda T)^{j-N}}{(j-N) !}\left[G^{(j)}(K)-G^{(j+1)}(K)\right]-\left[G^{(N)}(K)-G^{(N+1)}(K)\right] \sum_{j=N}^{\infty} \frac{(\lambda T)^{j-N}}{(j-N) !} G^{(j)}(K)\right] \\
& =G^{(N)}(K) \sum_{j=N}^{\infty} \frac{(\lambda T)^{j-N}}{(j-N) !} G^{(j)}(K)[Q(j)-Q(N)]>0,
\end{aligned}
$$

which follows that $Q(N, T)$ increases strictly with $N$ to 1 . Differentiating $Q(N, T)$ with respect to $T$,

$$
\frac{\sum_{j=0}^{\infty}\left[(\lambda T)^{j} / j !\right]\left[G^{(j+N+1)}-G^{(j+N+2)}(K)\right]}{\sum_{j=0}^{\infty}\left[(\lambda T)^{j} / j !\right] G^{(j+N+1)}(K)}-\frac{\sum_{j=0}^{\infty}\left[(\lambda T)^{j} / j !\right]\left[G^{(j+N)}-G^{(j+N+1)}(K)\right]}{\sum_{j=0}^{\infty}\left[(\lambda T)^{j} / j !\right] G^{(j+N)}(K)}>0
$$

which follows that $Q(N, T)$ increases strictly with $T$ to 1 . 